\title{
Refinement of third body parameters and new photometric results for the early-type multiple system IU Aurigae ${ }^{\star}$
}

\author{
S. Özdemir ${ }^{1,2}$, P. Mayer $^{3}$, H. Drechsel ${ }^{4}$, O. Demircan ${ }^{1,2}$, and H. Ak ${ }^{5}$ \\ ${ }^{1}$ Çanakkale Onsekiz Mart University, Department of Physics, 17100 Çanakkale, Turkey \\ 2 Çanakkale Astrophysics Research Center, Terzioğlu Campus, 17100 Çanakkale, Turkey \\ 3 Astronomical Institute, Charles University, Švédská 8, 15000 Praha 5, Czech Republic \\ ${ }^{4}$ Dr. Remeis-Sternwarte, Astronomisches Institut der Universtät Erlangen-Nürnberg, Sternwartstrasse 7, \\ 96049 Bamberg, Germany \\ 5 Ankara University, Department of Astronomy and Space Sciences, 06100 Ankara, Turkey
}

Received 3 January 2003 / Accepted 7 March 2003

\begin{abstract}
The massive eclipsing binary system IU Aurigae is re-analyzed in the context of new photometric observations and an improved treatment of its long-term period changes (O-C diagram) derived from new times of minima. The best-fit of the O-C curve reveals that the third component is orbiting around the system with a period of 293.3 days in a highly eccentric orbit ( $e=0.62$ ). A solution of the most recent $U B V$ light curves obtained during 1997/1998 showed a third light contribution of $\approx 23 \%$ and continuation of the orbital plane precession of IU Aurigae. Since the revised mass of the third component deduced from the analysis of the $\mathrm{O}-\mathrm{C}$ curve of $m_{3}=14.2 M_{\odot}$ would imply a larger fraction of third light than suggested by the value of $23 \%$ derived from the light curve analysis, the third body is possibly composed of two components. A $q$-search test applied to the $U B V$ light curves yields a minimum $\chi^{2}$ value at $q=0.67 \pm 0.02$, which confirms previous photometric and spectroscopic solutions.
\end{abstract}

Key words. binaries: eclipsing - stars: early-type - stars: individual: IU Aurigae

\section{Introduction}

The eclipsing binary system IU Aurigae (HD 35652; HIP 25565) with moderately hot components (O9.5 V+B0.5 IV-V) is the best studied example of a small group of binaries, which are members of multiple systems and exhibit a nodal line rotation and corresponding secular changes of the depths of their eclipses. The precessional motion of the orbital plane is due to a gravitationally bound third component in a non-coplanar orbit. In the case of IU Aur the existence of a third body has long been known from spectroscopic studies and the investigation of its light-time effect (LITE; see e.g., Mayer 1983 and Drechsel et al. 1994, hereafter DHLM). The third body orbits around the eclipsing binary in 293 days and causes the nodal line of the eclipsing pair to rotate with a period of about 335 years (DHLM). The system was monitored occasionally by various authors since it was discovered by Mayer (1965) in 1964 (period 1.8114, maximum $\mathrm{V}$ brightness 8.22 ). The analysis of previous light

Send offprint requests to: S. Özdemir,

e-mail: ozdemir@comu.edu.tr

* Based on the first observational data of a new optical observatory in Turkey, Çanakkale Onsekiz Mart University Observatory, operated by the Çanakkale Astrophysics Research Center (ÇAAM), URL:http://physics. comu. edu.tr/caam.html curves lead to a semi-detached configuration with a Roche lobe filling secondary (Mayer \& Drechsel 1987, DHLM). A review of the system and references to previous investigations of IU Aur can be found in DHLM.

Various attempts were performed to solve the orbital parameters of the third component. Mayer $(1983,1990)$ and Pettersen (1979) were the first to postulate the existence of a gravitationally bound third component due to the observed light time effect. Later spectroscopic and photometric studies also confirmed the third body, which contributes about $15-25 \%$ of the total light (Eaton 1978, DHLM). The precessional motion of the binary plane caused by the third component leads to a continuous change of the orbital inclination at a rate of about 0.5 per year during the last few decades (Mayer \& Drechsel 1987).

In a recent spectroscopic study, Harries et al. (1998) reported no sign of the third body. They used a cross-correlation method to derive a revised mass ratio of the eclipsing system of $q \approx 0.5$, which is much smaller than other determinations ( $q=$ $0.68 \pm 0.05$, see Mammano et al. 1977 or DHLM). However, this discrepant value can possibly be attributed to the crosscorrelation reduction technique used in their investigation, which is probably not adequate to resolve the phase-dependent third body contribution to the line profiles, even though their 
spectra have higher resolution than those of Mammano et al. (1977).

A visual component of IU Aur was discovered by HIPPARCOS (ESA 1997) and ICCD speckle interferometry (Mason et al. 1999, 1998), with a separation of $130 \pm 46$ mas (epoch 1991.25), 121: mas (epoch 1993.20), and 147 mas (epoch 1996.87), respectively. As estimated by Mason et al. (1998), the probable orbital period of the visual component is 430 years, i.e., the visual component cannot be the third body affecting the eclipsing binary inclination. Unfortunately, the magnitude difference of the visual component was determined by HIPPARCOS with rather large uncertainty: 1 . $36 \pm 1$. 01 . The fractional contributions of the third body in the triple system and the visual component to the observed extra light can therefore only be estimated.

In the present study the orbital parameters of the third body were refined by an analysis of all accurate minimum times collected from literature, complemented by new photoelectric observations. In addition, the most recent $U B V$ light curves obtained in 1997/1998 at Ankara University Observatory (AUO) and a few additional observations collected in 2002 at Çanakkale Onsekiz Mart University Observatory (ÇOMUO) were re-analyzed to monitor the continued inclination change of the system.

\section{Observational data}

New photometric observations were made on 13 nights at AUO in 1997/1998 and 2 nights at ÇOMUO in 2002 by using a $30 \mathrm{~cm}$ Cassegrain-Maksutov telescope and a $40 \mathrm{~cm}$ CassegrainSchmidt telescope, respectively. The detectors used in both observatories were single-channel, uncooled SSP-5 photometers equipped with Hamamatsu R1414 (at AUO) and R6358 (at ÇOMUO) photomultiplier tubes. The ÇOMUO observations are among the first photometric results of this observatory, which started to operate at the beginning of 2002.

The comparison and check stars were HD 35619 (SAO 58048) and HD 35633 (SAO 58053), respectively. In total, 966, 959 and 947 measurements were obtained in $U$, $B$ and $V$ filters, respectively, during the $1997 / 98$ season. The phasing of the light curves was performed using an updated linear ephemeris derived from inclusion of our 1997/98 observations:

HJD $($ prim. min. $)=2450737.451(5)+1.8114753(6) \times \mathrm{E}$.

The calculation of fluxes outside the Earth's atmosphere was made following the standard procedure described by Hardie (1962).

The differential measurements were made in a sequence comparison-variable-sky-variable-comparison. Total integration time in each filter was $15 \mathrm{~s}$. Standard deviations of the AUO observations determined from check minus comparison star measurements are $0.014,0.012$, and 0.018 in $U B V$ filters, respectively. Although the transformation coefficients of the $30 \mathrm{~cm}$ Cassegrain-Maksutov telescope of AUO are known to be $s=0.0056 \pm 0.0032, t=1.033 \pm 0.002$, and $w=$ $0.968 \pm 0.015$, transformation into standard magnitudes was not
Table 1. Log of photometric observations of IU Aur. Johnson $U B V$ and $B V R$ filters were used at Ankara University Observatory (AUO) and at Çanakkale Onsekiz Mart University Observatory (ÇOMUO), respectively.

\begin{tabular}{ccc}
\hline \hline $\begin{array}{c}\text { Start - stop } \\
(\mathrm{HJD}-2400000)\end{array}$ & $\begin{array}{c}\text { Phase } \\
\text { interval }\end{array}$ & $\begin{array}{c}\text { Date } \\
\text { dd/mm/yy }\end{array}$ \\
\hline $50737.3368-50737.6059$ & $0.93-0.08$ & $15 / 10 / 97$ \\
$50766.2572-50766.3722$ & $0.90-0.96$ & $13 / 11 / 97$ \\
$50776.3456-50776.3670$ & $0.47-0.48$ & $23 / 11 / 97$ \\
$50802.2647-50802.4485$ & $0.78-0.88$ & $19 / 12 / 97$ \\
$50818.1733-50818.3777$ & $0.56-0.67$ & $04 / 01 / 98$ \\
$50859.2553-50859.4604$ & $0.24-0.35$ & $14 / 02 / 98$ \\
$50866.2152-50866.4105$ & $0.08-0.19$ & $21 / 02 / 98$ \\
$50868.2143-50868.3743$ & $0.18-0.27$ & $23 / 02 / 98$ \\
$50870.3007-50870.4279$ & $0.34-0.41$ & $25 / 02 / 98$ \\
$50871.2170-50871.3830$ & $0.84-0.93$ & $26 / 02 / 98$ \\
$50872.2373-50872.4467$ & $0.40-0.52$ & $27 / 02 / 98$ \\
$50874.2383-50874.3812$ & $0.51-0.59$ & $01 / 03 / 98$ \\
$50876.3022-50876.4368$ & $0.65-0.72$ & $03 / 03 / 98$ \\
$52313.3066-52313.5150$ & $0.93-0.04$ & $07 / 02 / 02$ \\
$52319.2291-52319.5084$ & $0.20-0.35$ & $13 / 02 / 02$ \\
\hline
\end{tabular}

carried out, because no standard star observations had been performed (for details of standard magnitude transformation see Hardie 1962).

For the ÇOMUO observations, standard deviations calculated from a linear atmospheric extinction law are 0.020 , 0 . 014 , and 0.016 in $B V R$ filters, respectively, and may give an idea about the quality of the observational nights. Use of small telescopes and uncooled single channel photometers are the main reasons of the large scatter seen in the light curves. The log of observations is given in Table 1.

Two minimum times from the 1997/98 observations and one minimum time from the 2002 observations were derived by using the algorithm described by Kwee \& van Woerden (1956). These minimum times are listed in Table 2 together with the minimum depths, which are derived as the amplitude of the light curve between phase intervals with a width of \pm 0.015 centered on quadrature and eclipse minimum phases.

Since the LITE has a short period (293 days) in the case of IU Aur, the light curves suffer from a phase shift, if seasonal photometric observations cover a large fraction of LITE's period (see, e.g., Ohmori's light curves for an illustration of this effect, Ohmori 1991). Therefore, a correction term was included accordingly to avoid such phase shifting when constructing the light curves.

If the minimum depths listed in Table 2 are combined with those of DHLM (see their Fig. 3, in which the variation of the observed minimum depths were graphed versus time), the continued orbital plane precession of IU Aur is clearly documented. The minimum depths vs. time dependence is re-sketched in Fig. 1. Minimum depths estimated from photometry by HIPPARCOS (primary minimum depth 0.65 , secondary 0.47 , both for epoch JD 2448500 ) are also plotted. The previous prediction for the time of maximum inclination passage by DHLM (at 1984.3 \pm 0.3 ) is confirmed. 
Table 2. New minimum times of IU Aur observed at AUO and ÇOMUO. Secondary minimum was not used in calculation of third body orbit.

\begin{tabular}{cclc}
\hline \hline $\begin{array}{c}\text { Times of minima } \\
(\text { HJD-2400000) }\end{array}$ & Filter & Pri./sec. & Minimum depths \\
\hline $50737.4511 \pm 0.0005$ & $U$ & Pri. & $0.569 \pm 0.027$ \\
$50737.4523 \pm 0.0005$ & $B$ & Pri. & $0.529 \pm 0.026$ \\
$50737.4520 \pm 0.0004$ & $V$ & Pri. & $0.522 \pm 0.028$ \\
$50737.4518 \pm 0.0008^{a}$ & $U B V$ & Pri. & \\
$50872.3973 \pm 0.0004$ & $U$ & Sec. & $0.423 \pm 0.029$ \\
$50872.3942 \pm 0.0006$ & $B$ & Sec. & $0.413 \pm 0.029$ \\
$50872.3950 \pm 0.0006$ & $V$ & Sec. & $0.399 \pm 0.029$ \\
$50872.3955 \pm 0.0009^{a}$ & $U B V$ & Sec. & \\
$52313.4258 \pm 0.0016$ & $B$ & Pri. & $0.489 \pm 0.027$ \\
$52313.4252 \pm 0.0012$ & $V$ & Pri. & $0.484 \pm 0.019$ \\
$52313.4276 \pm 0.0007$ & $R$ & Pri. & $0.473 \pm 0.020$ \\
$52313.4262 \pm 0.0021^{a}$ & $B V R$ & Pri. & \\
\hline
\end{tabular}

${ }^{a}$ averaged value.

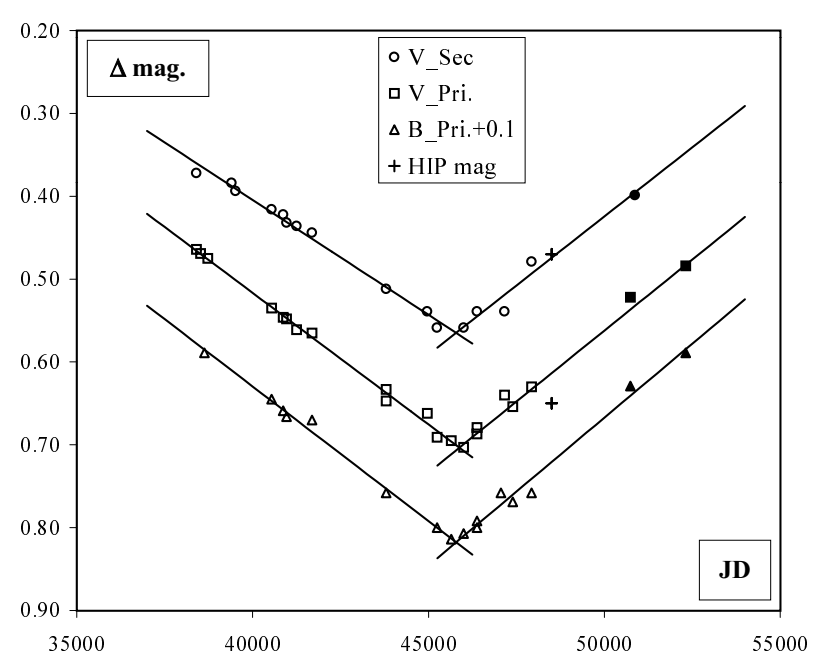

Fig. 1. Variation of minimum depths of IU Aur with time between 1964 and 2002. Best linear fits (solid lines) are shown. The filled symbols are the result of this work. The figure was adopted from DHLM and complemented by our new data and HIPPARCOS measurements.

\section{Analysis of the light curves}

The UBV light curves observed in 1997/98 (mean epoch 1998.0) were solved simultaneously with the MORO code (Drechsel et al. 1995), which is based on the conventional Wilson-Devinney approach. The MORO code takes into account the effects of radiation pressure, due to the mutual irradiation of early-type components of close binaries, and also uses a refined parameter optimization algorithm. The method was described in detail by Drechsel et al. (1995).

Original photometric data were binned into $\approx 0.01$ phase intervals to construct a less scattered light curve and to reduce the number of data points. A total of 118,117 , and 117 normal points representing the $U, B$, and $V$ filter measurements, respectively, were used as input data for the solution. Magnitudes were expressed as relative intensities and normalized to unity at the light level of first quadrature.
The initial values of the system parameters for the simplex iterations were taken from Mayer \& Drechsel (1987). Some parameters were fixed at their reliable theoretical values, such as gravity darkening exponents $\left(g_{1,2}=1.0\right)$ and albedos $\left(A_{1,2}=1.0\right)$. In accordance with all previous solutions it was assumed that the binary orbit is circular $(e=0)$, and the binary components are locked in a synchronous rotation. The limb darkening coefficients were adopted from Díaz-Cordovés et al. (1995). According to the spectral type of the primary $(09.5 \mathrm{~V})$, its effective temperature $T_{1}$ was fixed at $32000 \mathrm{~K}$. For all solution runs, Wilson-Devinney mode 2 was used, which means that no a-priori restriction of the system configuration was forced. The mass ratio was used as a free parameter. We performed a large number of trial solutions for various different start values of $q$ between 0.50 and 0.90 . It turned out that convergent solutions with reasonably small standard deviations confined $q$ to the range $0.65-0.75$. A minimum $\chi^{2}$ was found at $q=0.67 \pm 0.02$, which confirms the spectroscopic results of Mammano et al. (1977) and DHLM, who found $q=0.68 \pm 0.05$.

Table 3 gives the final parameters of the current photometric solution of the $1997 / 98 U B V$ curves. A previous solution of IU Aur light curves of 1984.4 by DHLM is also included in Table 3 for comparison. The inclination change with time due to gravitational effects of the third component is evident from the resulting $i$ values of $90^{\circ} .0 \pm 0.3$ and $83^{\circ} .7 \pm 0.8$, respectively. As in all previous solutions again a semi-detached configuration with a Roche-lobe filling secondary component is found. A 3-dimensional representation of the binary surfaces is depicted in Fig. 2. The third light contribution in the light curves came out as $19.8 \%, 24.3 \%$, and $24.0 \%$ in $U, B$, and $V$ filters, respectively. This result is in accordance with the solutions of earlier light curves of 1964, 1973, 1974 and 1984 by DHLM. The light curves and theoretical curves derived from the solution parameters listed in Table 3 are shown in Fig. 3.

\section{Third body orbit}

Parameters of the third body orbit can be derived from a solution of the $\mathrm{O}-\mathrm{C}$ curve. In the case of IU Aur, besides the geometrical light-time effect, the dynamical term described by, e.g., Söderhjelm (1975, hereafter S75), also has to be accounted for when fitting the $\mathrm{O}-\mathrm{C}$ residuals. Its semi-amplitude reaches 0.0015 , and hence causes a non-negligible contribution.

The value of inclination found in the previous section agrees well with an extrapolation of the long-term inclination change given by DHLM (see their Fig. 5), who fitted the observations with the theoretical variation of $i$ expected for the precessional motion of the eclipsing binary plane in a triple system. Therefore the following calculations are based on values derived from the solution presented in that paper, which are adopted as valid for epoch 1984.4, in spite of their preliminary character. So we assume $P_{\text {node }}=335$ years, $i_{1}^{0}=90^{\circ}$, $I=85^{\circ} .9, i_{1}=29^{\circ} .0, m_{0}=21.4, m_{1}=14.3 M_{\odot}$. The notation used follows S75, i.e., $i_{1}^{0}$ is the inclination angle of the eclipsing system, $I$ is the inclination of the invariant plane against the observer's celestial plane, and $i_{1}$ is the angle between the 


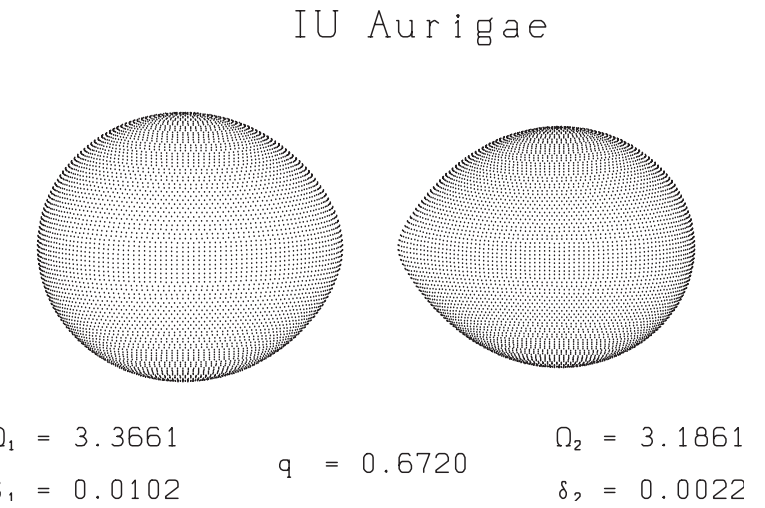

Fig. 2. 3-D aspect of the IU Aur binary components at first quadrature. The cooler secondary essentially fills its critical Roche lobe.

invariant plane of the triple system and the orbital plane of the eclipsing system, while $m_{0}, m_{1}$ and $m_{2}$ represent the masses of the eclipsing binary components, and the mass of the third body, respectively.

The first calculation including the dynamical term yielded a third body mass $m_{2}$ of about $15 M_{\odot}$; then the ratio of orbital moments is $G_{2} / G_{1}=\sin i_{1} / \sin i_{2}=7.5$ (see S75, Eq. (20)). Therefore $i_{2}=4.0$, and the mutual inclination of both orbital planes equals $j=33^{\circ}$.0. These values were taken as initial parameters and allowed to change during subsequent iterations of our numerical solution procedure.

The published times of minima cover the time interval from 1964 to 2002 . We used only photoelectrically measured times of minima as compiled by Mayer (1987) and newer minima from various sources with errors estimated to be smaller than 0.0020. All measurements were given identical weight (the table of minima is available on request).

Since the orbital planes rotate such that the longitude of the node $\Omega$ (see S75, Fig. 2) decreases by $360 / 335$ degrees per year, one has to account for the change of the geometrical argument of periastron (counted from the sky plane), $\omega^{0}$, during the mentioned time interval. The argument of periastron with respect to the fundamental plane, $\omega$, is changing as well, but considerably slower (S75, Eq. (36)), so that it can be assumed as constant during the considered period of time.

Therefore,

$\Omega=\Omega_{1984.3}-b\left(J D-J D_{1984.3}\right)$

where $J D_{1984.3}=2445800$, the change of $\Omega$ per day is $b=$ 0.00294 and

$\cos \Omega_{1984.3}=\cot I \cot i_{1} ;$

numerically, $\Omega_{1984.3}=82$.6. Furthermore, $\omega^{0}=\omega+s_{2}$, and

$\sin s_{2}=\sin I \sin \Omega / \sin i_{2}$.

The geometrical effect as frequently given in the literature (see, e.g., Irwin 1959; Mayer 1990) is described by

$$
\begin{aligned}
\mathrm{O}-\mathrm{C}_{\mathrm{geom}} & =t-\left(t_{0}+P_{1} E\right) \\
& =\frac{a_{12} \sin i^{0}}{173.14}\left[\frac{1-e^{2}}{1+e \cos v} \sin \left(v+\omega^{0}\right)+e \sin \omega^{0}\right]
\end{aligned}
$$

Table 3. Simultaneous MORO solution of the $U B V$ light curves of the eclipsing system IU Aur; for comparison, a previous solution is also presented in the third column.

\begin{tabular}{lcc}
\hline \hline Parameter & this paper & DHLM \\
\hline Fixed parameters & & \\
\hline$A_{1}=A_{2}{ }^{a}$ & 1.0 & 1.0 \\
$g_{1}=g_{2}{ }^{b}$ & 1.0 & 1.0 \\
$x_{1}(U)^{c}$ & 0.342 & 0.20 \\
$x_{1}(B)^{c}$ & 0.337 & 0.25 \\
$x_{1}(V)^{c}$ & 0.303 & 0.20 \\
$x_{2}(U)^{c}$ & 0.353 & 0.30 \\
$x_{2}(B)^{c}$ & 0.352 & 0.35 \\
$x_{2}(V)^{c}$ & 0.313 & 0.30 \\
$T_{1}(\mathrm{~K})$ & 32000 & 32000 \\
\hline
\end{tabular}

\begin{tabular}{lcc}
\hline \multicolumn{3}{l}{ Adjusted parameters } \\
\hline$i$ & $\begin{array}{c}83.7 \pm 0.8 \\
\text { (epoch } 1998.0)\end{array}$ & $\begin{array}{c}90.0 \pm 0.3 \\
\text { (epoch } 1984.4)\end{array}$ \\
$q\left(=M_{2} / M_{1}\right)$ & $0.672 \pm 0.017$ & 0.691 \\
$T_{2}(\mathrm{~K})$ & $27540 \pm 290$ & 28220 \\
$\Omega_{1}$ & $3.366 \pm 0.032$ & 3.502 \\
$\Omega_{2}$ & $3.186 \pm 0.037$ & 3.212 \\
$L_{1}(U)^{d}$ & $0.625 \pm 0.007$ & 0.587 \\
$L_{1}(B)^{d}$ & $0.617 \pm 0.008$ & 0.580 \\
$L_{1}(V)^{d}$ & $0.610 \pm 0.007$ & 0.574 \\
$l_{3}(U)^{e}$ & $19.8 \% \pm 1.8 \%$ & $16.9 \%$ \\
$l_{3}(B)^{e}$ & $24.3 \% \pm 1.6 \%$ & $20.8 \%$ \\
$l_{3}(V)^{e}$ & $24.0 \% \pm 1.7 \%$ & $21.1 \%$ \\
$\delta_{1}{ }^{f}$ & $0.010 \pm 0.004$ & 0.012 \\
$\delta_{2}{ }^{f}$ & $0.002 \pm 0.002$ & 0.001 \\
\hline Roche radii ${ }^{g}$ & & \\
\hline$r_{1}$ (pole) $^{\circ}$ & $0.362 \pm 0.006$ & 0.348 \\
$r_{1}$ (side) & $0.378 \pm 0.009$ & 0.362 \\
$r_{1}$ (back) & $0.398 \pm 0.009$ & 0.379 \\
$r_{1}$ (point) & $0.423 \pm 0.008$ & 0.396 \\
$r_{2}$ (pole) & $0.324 \pm 0.003$ & 0.327 \\
$r_{2}$ (side) & $0.339 \pm 0.004$ & 0.343 \\
$r_{2}$ (back) & $0.371 \pm 0.005$ & 0.376 \\
$r_{2}$ (point) & $0.425 \pm 0.014$ & 0.435 \\
\hline $1 \sigma$ deviation & 0.0074 & 0.0039 \\
\hline
\end{tabular}

${ }^{a}$ Bolometric albedo.

${ }^{b}$ Gravitational darkening exponent.

${ }^{c}$ Linear limb darkening coefficient; theoretical values taken from Díaz-Cordovés et al. (1995).

${ }^{d}$ Relative luminosities $L_{i} /\left(L_{1}+L_{2}\right) ; L_{2}$ is not independently adjusted, but recomputed from $r_{2}$ and $T_{2}$.

${ }^{e}$ Fraction of third light at maximum.

${ }^{f}$ Radiation pressure parameter, see Drechsel et al. (1995).

${ }^{g}$ Fractional Roche radii in units of separation of mass centers.

where $P_{1}$ is the apparent mean period of the eclipsing system and $a_{12}, v, e, \omega^{0}$ and $i^{0}$ are the parameters of the eclipsing binary orbit around the common center of mass of the triple system.

The mass function of the triple system is given by:

$$
\begin{gathered}
f\left(m_{2}\right)=\frac{\left(a_{12} \sin i^{0}\right)^{3}}{P_{2}^{2}}=\frac{\left(m_{3} \sin i^{0}\right)^{3}}{\left(m_{0}+m_{1}+m_{2}\right)^{2}} \\
=\frac{1}{P_{2}^{2}}\left[\frac{c^{2} A^{2}}{\left(1-e^{2} \cos ^{2} \omega^{0}\right)}\right]^{3 / 2},
\end{gathered}
$$




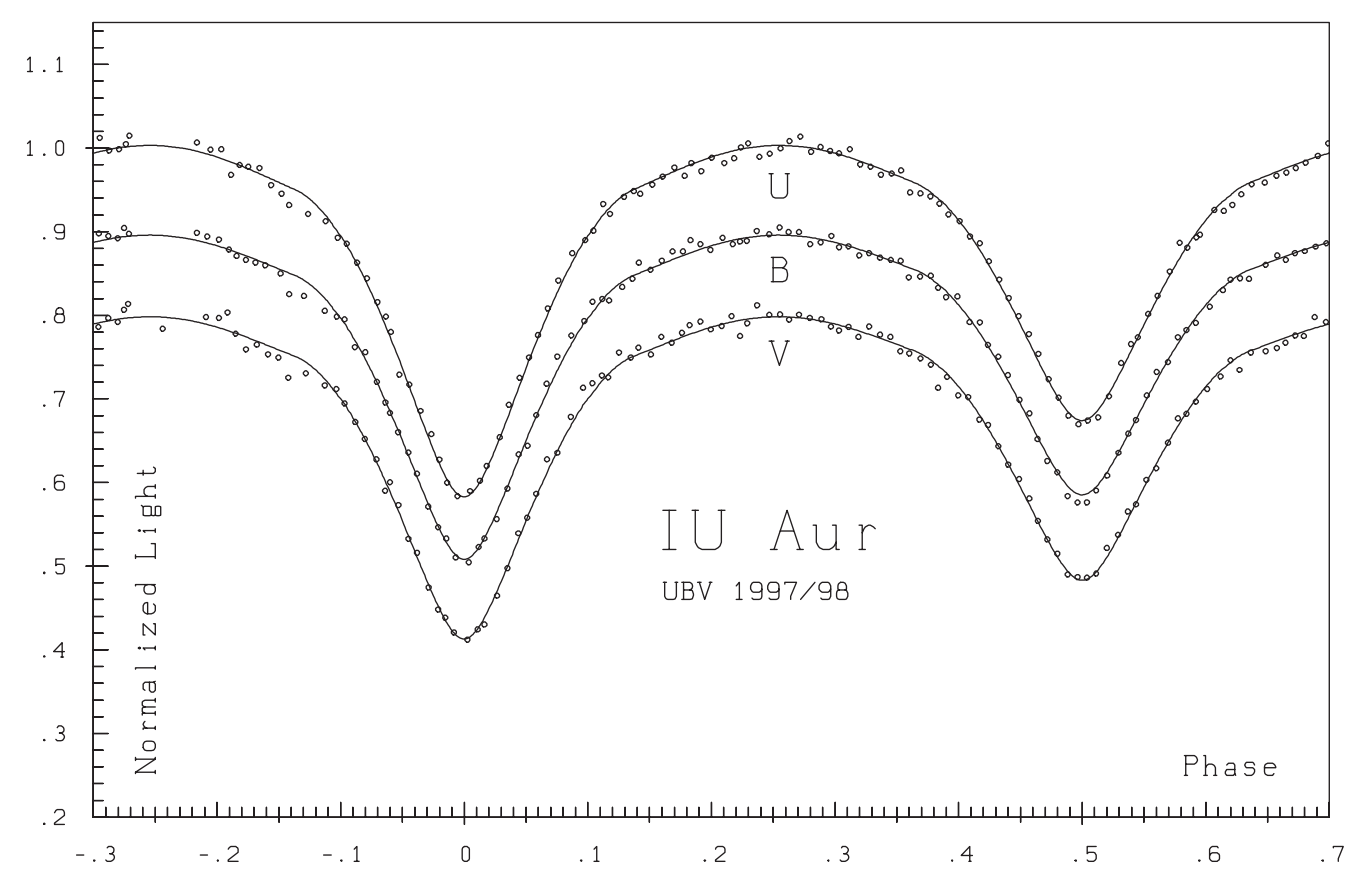

Fig. 3. Observed normalized $U B V$ light curves obtained in 1997/98 (dots) and best fit theoretical curves (solid lines) of IU Aur. The curves are given in intensity units, while $B$ and $V$ data are offset by 0.1 each. Phases were calculated according to the ephemeris of Eq. (1).

where $P_{2}$ is the orbital period of the third component. The observed semi-amplitude of the LITE, $A$, is given by

$A=\frac{a_{12} \sin i^{0} \sqrt{\left(1-e^{2} \cos ^{2} \omega^{0}\right)}}{173.14}$.

In order to solve for the orbital parameters, we used the analytical form of the dynamical contribution to $\mathrm{O}-\mathrm{C}$ as given by Mayer (1990) and corrected by Borkovits et al. (2003):

$$
\begin{aligned}
\mathrm{O}-\mathrm{C}_{\mathrm{dyn}}= & \frac{3}{8 \pi} \frac{m_{2}}{m_{0}+m_{1}+m_{2}} \frac{P_{1}^{2}}{P_{2}}\left(1-e^{2}\right)^{-\frac{3}{2}} \\
& \times\left[(2-Z) W+\left(Z-\frac{2}{3}\right)(v-l+e \sin v)\right]
\end{aligned}
$$

where

$W=\sin 2(v+\omega)+e\left(\sin (v+2 \omega)+\frac{1}{3} \sin (3 v+2 \omega)\right)$

and $Z=\cos j+\cos ^{2} j ; l$ is the mean anomaly.

By applying an iterative procedure, we minimized the differences between the minimum times predicted by accounting for geometrical plus dynamical contributions and the measured minimum times. The results are given in Table 4 . The same process also allowed for a new determination of $m_{2}=14.2 M_{\odot}$, in accordance with the mass function and a reliable value of the orbital inclination derived from the analysis of light curves of epoch $1984.4 ; i_{1}^{0}=90^{\circ} .0$.

When the formula by Borkovits et al. (2003, their formula (36)) is used, then the amplitude of the dynamical term reaches only 0.0012 , i.e., the amplitude of the geometrical term is larger and the mass of the third body is $15 M_{\odot}$. The other parameters change only insignificantly.

The observed $\mathrm{O}-\mathrm{C}$ values folded by the long-period given in Table 4 are shown in Fig. 4 (upper part) together with the curve for the dynamical term. Since $\omega^{0}$ is different for each time of measurement, no unique curve representing the geometrical term exists. But we plotted curves valid for two epochs close to the beginning and end of the interval with observations: one for the year $1964\left(\omega^{0}=24^{\circ}\right)$, another for 2000 $\left(\omega^{0}=-16^{\circ}\right)$. Differences between observations and theoretical expressions $\mathrm{O}-\mathrm{C}_{\mathrm{geom}}+\mathrm{O}-\mathrm{C}_{\mathrm{dyn}}$ are shown in the lower part of the figure.

\section{Discussion}

The solution of the $1997 / 98$ light curves proves that: i) The changing of minimum depths is continuing as expected. The inclination computed for 1997/98 (i.e., $i=83.7 \pm 0.8$ ) is well compatible with the prediction of DHLM's Fig. 5 in which cosine of inclination is plotted versus time; ii) The amount of third light (on average $23 \%$ for $U B V$ filters) is consistent with previous calculations based on both photometry and spectroscopy (Eaton 1978, DHLM). Such a level of light contamination would correspond to a less massive third body than suggested by the value of $m_{3}=14.2 M_{\odot}$ following from the O-C analysis, if it is assumed to be a main sequence star. Therefore we propose that the third body responsible for the extra light in the light curves is not a single, but a double star. A precise analysis of the O-C curve increases the value of the eccentricity of the third body orbit to $e=0.62$, which is much larger than previous determinations (Pettersen 1979; Mayer 1983, 1990).

The corresponding absolute magnitude $M_{V}$ of the primary deduced from its spectral type of $09.5 \mathrm{~V}$ is about -4 . 1 (using $L_{1}$ calculated during the light curve solution one gets a nearly identical value); the total light of the system is more than twice as large, i.e., $M_{V \text {,total }}=-4 \mathrm{~m} .9$, and with $V=8.22$ in maximum and a color excess of $E(B-V)=0.53\left(A_{V}=1.64\right)$, the distance modulus is 11.5 which leads to a distance of $\approx 2 \mathrm{kpc}$. 


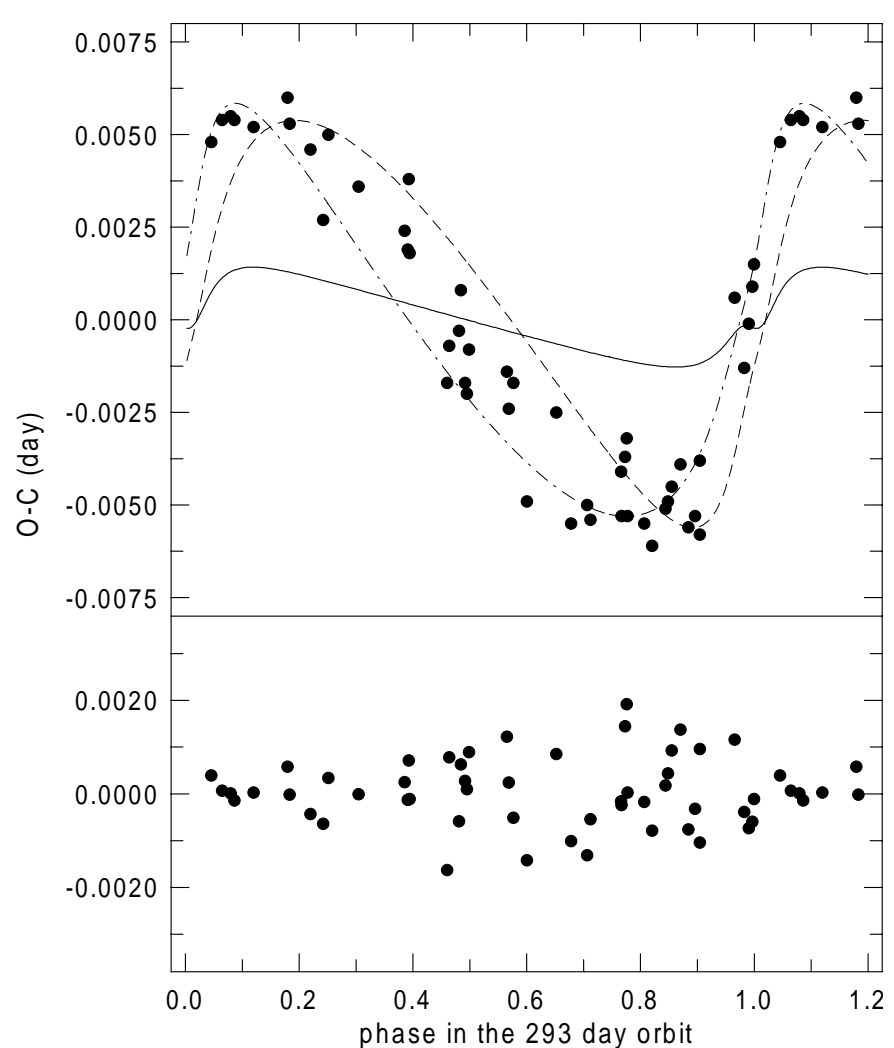

Fig. 4. Upper part: The observed $\mathrm{O}-\mathrm{C}_{\mathrm{lin}}$ versus phase of the long orbit; $C_{\text {lin }}=2438448.40705+1.8114742 . E$. The full line represents the dynamical term, dot-dashed line is valid for $\omega^{0}=24^{\circ}$, dashed line for $\omega^{0}=-16^{\circ}$. Lower part: Residuals relative to minimum times calculated using the sum of geometrical and dynamical terms.

Table 4. Third-body orbital parameters.

\begin{tabular}{lcc}
\hline \hline Parameter & Unit & Value \\
\hline$P_{1}$ & day & 1.8114741 \\
$H J D_{0}$ & & 2438448.4073 \\
$P_{2}$ & day & 293.3 \\
$J D_{\text {periastron }}$ & & 2438010 \\
$e$ & & 0.615 \\
$a_{1,2}$ & day & 0.00522 \\
$\omega_{1984.3}^{0}$ & deg & 2.7 \\
$m_{2}$ & $M_{\odot}$ & 14.2 \\
$r m s$ of an $\mathrm{O}-\mathrm{C}$ & day & 0.00079 \\
\hline
\end{tabular}

Therefore, the IU Aur system might belong to the Aur OB2 association; note that Humphreys (1978) lists HD 35619 - the comparison star - as a member of this association, with an only slightly different modulus of 11.67. The HIPPARCOS trigonometric parallax of $221 \mathrm{pc}$ is certainly irrelevant and possibly affected by the multiple nature of the system.

Extensive trial runs with the MORO code yielded a number of convergent solutions of the $U B V$ light curves of comparable fit quality (i.e. with $1 \sigma$ deviations of observed fluxes from the theoretical light curves less than $5 \%$ worse than the value of $\sigma=0.0074$ of the best solution of Table 3). The errors of individual parameters quoted in this table are standard deviations corresponding to the scatter of parameters around their mean values calculated from the nine best solutions. The best
Table 5. Absolute dimensions of IU Aur according to the new photometric solution of Table 3 .

\begin{tabular}{lll}
\hline \hline Parameter & Primary & Secondary \\
\hline$M\left(M_{\odot}\right)$ & $21.4 \pm 2.5$ & $14.5 \pm 2$ \\
$R\left(R_{\odot}\right)$ & $7.8 \pm 0.8$ & $7.1 \pm 0.8$ \\
$T(\mathrm{~K})$ & 32000 & $27540 \pm 290$ \\
$\log (L)\left(L_{\odot}\right)$ & $4.76 \pm 0.04$ & $4.42 \pm 0.05$ \\
$a\left(R_{\odot}\right)^{1}$ & $8.3 \pm 0.2$ & $12.3 \pm 0.8$ \\
$<\rho>(\operatorname{cgs})$ & $0.06 \pm 0.05$ & $0.06 \pm 0.05$ \\
$\log g(\operatorname{cgs})$ & $3.99 \pm 0.17$ & $3.89 \pm 0.19$ \\
$M_{V}\left({ }^{\mathrm{m}}\right)$ & $-3.72 \pm 0.05$ & $-2.80 \pm 0.11$ \\
\hline
\end{tabular}

solution parameter set itself, however, is the result of the accepted best individual solution, and not the mean of a multitude of solutions. We felt that this way the uncertainties of parameters are estimated in a more realistic way than by giving any formal numerical fit errors. No acceptable solutions were achieved for values of the mass ratio $q$ outside the range $0.65-0.75$. The mean value from our nine best solutions is $0.67 \pm 0.02$, fully compatible with the final solution value of 0.672 . Therefore it can be concluded that the discrepant smaller value of $q=0.51$ given by Harries et al. (1998) in a study, which did not account for the third body lines, can be ruled out, while the older results of Mammano et al. (1977) $(q=0.68 \pm 0.05)$ and DHLM $(q=0.679 \pm 0.057)$ are confirmed.

The third light found in the system amounts to $24 \%$ of total light at quadrature in the $V$ region. If one would attribute this extra light to a single source, the magnitude difference would be 1.78 . Such a value would fit the magnitude difference of the visual component as suggested by the HIPPARCOS measurement, with no need to postulate any further light source in the system. However, we have to account for the fact that the extra light must also include the fraction of third light emitted by the physical third body of the triple system. Its contribution could be rather modest if we rely on the HIPPARCOS finding, which implies that the visual companion alone would be sufficiently luminous to account for the observed third light, although the error margin of the HIPPARCOS magnitude difference of $1.36 \pm 1.01$ is rather large.

If we assume that the third body is a single main-sequence star with a mass of $14.2 M_{\odot}$, its absolute visual magnitude should be about $M_{V}=-3.6$. This would correspond to a far too large fraction of the total light of the system of about $30 \%$. The third body might therefore be a binary itself. Lowest luminosity is expected if both components would be of identical mass. The luminosity corresponding to a star of $7.1 M_{\odot}$ is $M_{V}=-1.8$, and a pair of them would sum up to -2.5 mag or $12 \%$ of the luminosity of the eclipsing system. In this case the fraction of third light to be attributed to the visual companion would be almost covered, but is still at the lower limit of what is suggested by the HIPPARCOS result.

The possibility of the detection of a long-period perturbative effect of a distant third companion on the motion of a close binary was studied in detail by Borkovits et al. (2003). They report that there are a few known systems for which the amplitude of the dynamical term of $\mathrm{O}-\mathrm{C}$ exceeds significantly the 


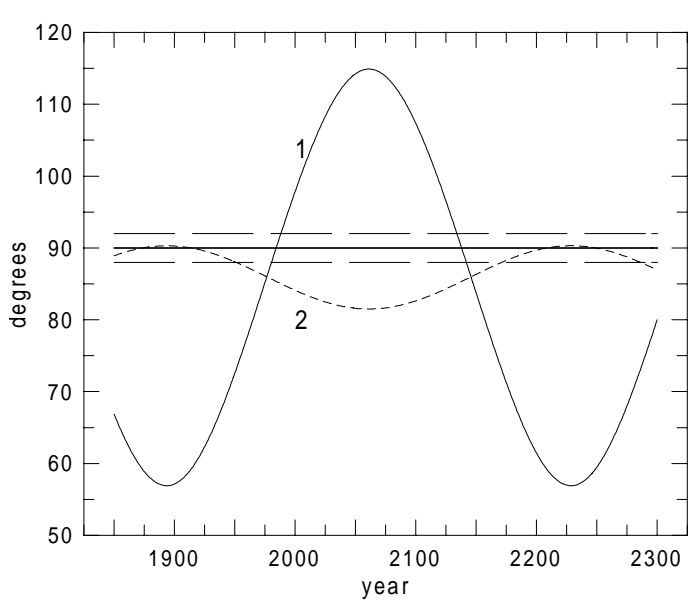

Fig. 5. Behavior of inclinations. 1: $i_{1}^{0}, 2: i_{2}^{0}$; long-dashed lines represent limits for mutual eclipses, when $i_{2}^{0}=88$ or $92^{\circ}$.

present observational accuracy. The IU Aur system is close to the limit of detectability of the dynamical contribution of its third body. An unambiguous distinction of this perturbative effect on the $\mathrm{O}-\mathrm{C}$ curve requires rather frequent and accurate minimum time observations, covering a full cycle of the third body revolution, i.e., at least about 300 days in the case of IU Aur.

The third body should also cause a change of $P_{1}$ during the nodal precession (see S75, Eq. (33)). We tried to find a change of $P_{1}$ during the iteration process (Sect. 4). However, no change was evident in the time interval 1964-2002 - residuals were always smallest for constant $P_{1}$.

We assumed the values of $I, i_{1}$ and $P_{2}$ found by DHLM as correct. With the present results, Söderhjelm's (S75) Eq. (27) gives a somewhat shorter nodal period, and also $I$ and $i_{1}$ would differ. However, given the observational and numerical limitations, the present results still have to be preliminary, and it appears appropriate to adopt the assumed values $(I=85.9$, $i_{1}=29$ and $i_{2} \approx 4^{\circ}$ ) also for the following considerations.

After the passage of maximum inclination in 1984, the depth of eclipses is now decreasing again and will continue to do so until about the year 2061 (see Fig. 5). Then eclipses are starting to deepen again and will be total in 2138 . Note the interesting behavior of $i_{2}^{0}$. It might be close to $90^{\circ}$ when $\Omega$ is close to zero. Then mutual eclipses of the third body and the eclipsing pair will appear. Under these conditions $i_{1}^{0} \approx 90-j$, i.e., $57^{\circ}$, and the semiminor axes equal about 4.5 and $6.7 R_{\odot}$. With $a_{12,3}=0.89 \mathrm{AU}=190 R_{\odot}$, total eclipses of the secondary might occur in the inclination range $90 \pm 2^{\circ}$, and of the primary within $90 \pm 1.4$. Unfortunately, the orientation of the system is such that these eclipses ended approximately 50 years ago, and will happen again only in the second half of the 22nd century to last for about one century. The eclipses will appear twice during the 293 d period in "windows" lasting up to 6 days.

It is undoubtable that the mutual eclipses would provide perfect information about the system. Hopefully, advanced interferometry will meet this aim already sooner, since the third body separation reaches about 2 mas.

Acknowledgements. This research was supported in part by the Scientific and Technical Research Council of Turkey (TÜBİTAK), and the Research Fund of Çanakkale Onsekiz Mart University. The authors thank Dr. T. Borkovits for sending a preprint version of their manuscript and for important remarks. The $40 \mathrm{~cm}$ Cassegrain-Schmidt telescope of the ÇOMUO was donated by OPTRONIK company.

\section{References}

Borkovits, T, Érdi, B., Forgács-Dajka, E., \& Kovács, T. 2003, A\&A, 398, 1091

Díaz-Cordovés, J, Claret, A., \& Giménez, A. 1995, A\&AS, 110, 329

Drechsel, H., Haas, S., Lorenz, R., \& Mayer, P. 1994, A\&A, 284, 853 (DHLM)

Drechsel, H., Haas, S., Lorenz, \& R., Gayler 1995, A\&A, 294, 723

ESA 1997, The Hipparcos \& Tycho Catalogues, ESA SP-1200

Eaton, J. A. 1978, Acta Astron., 28, 63

Hardie, E. 1962, in Astronomical Techniques: Stars and Stellar Systems, vol. II, ed. W. A. Hiltner (The University of Chicago Press)

Harries, T. J., Hilditch, R. W., \& Hill, G. 1998, MNRAS, 295, 386

Humphreys, R. M. 1978, ApJS, 38, 309

Irwin, J. B. 1959, AJ, 64, 149

Kwee, K. K., \& van Woerden, H. 1956, Bull. Astr. Int. Neth., 12, 327

Mammano, A., Margoni, R., \& Stagni, R. 1977, A\&A, 59, 9

Mason, B. D., Gies, D. R., Hartkopf, W. I., et al. 1998, AJ, 115, 821

Mason, B. D., Martin, C., Hartkopf, W. I., et al. 1999, AJ, 117, 1890

Mayer, P. 1965, PASP, 77, 436

Mayer, P. 1983, Bull. Astron. Inst. Czech., 34, 335

Mayer, P. 1987, Bull. Astron. Inst. Czech., 38, 58

Mayer, P., \& Drechsel, H. 1987, A\&A, 183, 61

Mayer, P. 1990, Bull. Astron. Inst. Czech., 41, 231

Ohmori, S. 1991, IBVS, 3674

Pettersen, B. R. 1979, A\&A, 80, 265

Söderhjelm, S. 1975, A\&A, 42, 229 (S75) 